\title{
Long-term evolution of an ecosystem with spontaneous periodicity of mass extinctions
}

\author{
AdAm LipowsKi $^{1}$ and Dorota Lipowska ${ }^{2}$ \\ ${ }^{1}$ Faculty of Physics, Adam Mickiewicz University, \\ 61-614 Poznań, Poland \\ ${ }^{2}$ Institute of Linguistics, Adam Mickiewicz University, \\ 60-371 Poznań, Poland
}

\begin{abstract}
Twenty years ago, after analysing palaeontological data, Raup and Sepkoski suggested that mass extinctions on Earth appear cyclically in time with a period of approximately 26 million years (My). To explain the $26 \mathrm{My}$ period, a number of proposals were made involving, e.g., astronomical effects, increased volcanic activity, or the Earth's magnetic field reversal, none of which, however, has been confirmed. Here we study a spatially extended discrete model of an ecosystem and show that the periodicity of mass extinctions might be a natural feature of the ecosystem's dynamics and not the result of a periodic external perturbation. In our model, periodic changes of the diversity of an ecosystem and some of its other characteristics are induced by the coevolution of species. In agreement with some palaeontological data, our results show that the longevity of a species depends on the evolutionary stage at which the species is created. Possible further tests of our model are also discussed.
\end{abstract}

Keywords: prey-predator lattice systems, simulations

\section{Introduction}

Recently, numerous efforts are being made to describe the large-scale evolution of the Earth ecosystem. Due to the ecosystem's complexity, however, such a task is extremely difficult (Pimm, 2002). That is why researchers in this field have to turn to very simplified and abstract models that hopefully still contain relevant factors. Such an approach proved to be successful, e.g., in modelling of some aspects of extinction dynamics (Newman \& Palmer, 2003). Indeed, in very simple models of ecosystems certain properties of extinctions as, e.g., the distribution of sizes or durations of extinctions, seem to agree, at least qualitatively, with palaeontological data (Bak \& Sneppen, 1993; Solé \& Manrubia, 1999). In these models, the dynamics spontaneously drives the ecosystem toward the scale-invariant state with extinctions described by some power-law characteristics. However, since the accuracy of fossil data is rather limited, especially with respect to events on a large timescale, the applicability of such models should be considered with care.

The suggestion that the extinction dynamics is not scale invariant but it has a characteristic timescale was made by Raup and Sepkoski (Raup \& Sepkoski, 1984). While analysing fossil data, they noticed that during the last 250 My mass extinctions on Earth appeared more or less cyclically with a period of approximately $26 \mathrm{My}$. Although their analysis was initially questioned (Patterson \& Smith, 1989), some other works confirmed Raup and Sepkoski's hypothesis (Fox, 1987; Prokoph et al., 2000; Plotnick \& Sepkoski, 2001). The suggested large periodicity of 
mass extinctions turned out to be very difficult to explain. Indeed, $26 \mathrm{My}$ does not seem to match any of known Earth cycles and some researchers have been looking for more exotic explanations involving astronomical effects (Davies et al., 1984; Rampino \& Stothers, 1984), increased volcanic activity (Stothers, 1993), or the Earth's magnetic field reversal (Stothers, 1986). So far, however, none of these proposals has been confirmed. One should also note that the most recent analysis of palaeontological data that span last 542My strongly supports the periodicity of mass extinctions albeit with a larger cycle of about 62My (Rohde \& Muller, 2005).

Lacking a firm evidence of an exogenous cause, one can ask whether the periodicity of extinctions can be explained without referring to such a factor. It is already well known that a periodic behaviour of a system is not necessarily the result of periodic driving. In particular, since the seminal works of Lotka and Volterra, it is known that spontaneous oscillations of the population size might appear in various prey-predator systems (Murray, 1989). However, the period of oscillations in such systems is determined by the growth and death rate coefficients of interacting species and is of the order of a few years rather than tens of millions. Consequently, if the periodicity of mass extinctions is to be explained within a model of interacting species, a different mechanism that generates long-period oscillations must be at work.

Recently, a multi-species prey-predator model has been introduced, where long-term oscillatory behaviour is observed (Lipowski, 2005). Only some preliminary studies of basic properties of this model have been made, and the objective of the present paper is to provide its more detailed analysis. In this model the period of oscillations is determined by the inverse of the mutation rate and as we argue, it should be several orders of magnitude longer than in the Lotka-Volterra oscillations. The mechanism that generates oscillations in our model can be briefly described as follows: A coevolution of predator species induced by the competition for food and space causes a gradual increase of their size. However, such an increase leads to the overpopulation of large predators and a shortage of preys. It is then followed by a depletion of large species and a subsequent return to the multi-species stage with mainly small species that again gradually increase their size and the cycle repeats. Numerical calculations for our model show that the longevity of a species depends on the evolutionary stage at which the species is created. A similar pattern has been observed in some palaeontological data (Miller \& Foote, 2003) and, to our knowledge, the presented model is the first one that reproduces such a dependence. Let us notice that the oscillatory behaviour in a prey-predator system that was also attributed to the coevolution has been already examined by Dieckmann et al. (Dieckmann et al., 1995). In their model, however, the number of species is kept constant and it cannot be applied to study extinctions. Moreover, the idea that an internal ecosystem dynamics might be partially responsible for the long-term periodicity in the fossil records was suggested by Stanley (Stanley, 1990) and later examined by Plotnick and McKinney (Plotnick \& McKinney, 2001). However, in his approach mass extinctions are triggered by external impacts. Their approximately equidistant separation is the result of a delayed recovery of the ecosystem. In our approach no external factor is needed to trigger such extinctions and sustain their approximate periodicity.

\section{Model}

Numerical simulations and models of various levels of description have been frequently used to study extinctions of species (Newman \& Palmer, 2003). In the simplest cases, the dynamics of models was formulated at the level of species and had to refer to the notion of fitness that is not commonly accepted. In more recent approaches, an individual-oriented dynamics has often been used and although computationally more demanding, such models are considered as more adequate (Caldarelli et al., 1998; Chowdhury et al., 2003; Coppex et al., 2004). Our model uses the individual-oriented dynamics but in addition it is spatially extended. Such a feature increases the computational complexity even more but it also takes into account, e.g., 
dynamically generated spatial inhomogeneities that sometimes are known to play an important role. Our model can be also considered as a multi-species generalization of the already studied spatially extended prey-predator model (Lipowski, 1999; Lipowski and Lipowska, 2000). Some other multi-species lattice models were also studied in various contexts (Pȩkalski, 2004; Sato et al., 2002; Dieckmann et al., 2000).

Our model describes a multi-species prey-predator system defined on a square lattice of linear size $N$ (Lipowski, 2005). At each site of a lattice $i$ there is an operator $x_{i}$ that specifies whether this site is occupied by a prey $\left(x_{i}=1\right)$, by a predator $\left(x_{i}=2\right)$, by both of them $\left(x_{i}=3\right)$, or is empty $\left(x_{i}=0\right)$. Each predator is characterized by its size $m(0<m<1)$ that determines its consumption rate and at the same time its strength when it competes with other predators. Only approximately the size $m$ can be considered as related with physical size. Predators and preys evolve according to rules typical to such systems (e.g., predators must eat preys to survive, preys and predators can breed provided that there is an empty site nearby, etc.). In addition, the relative update rate for preys and predators is specified by the parameter $r(0<r<1)$ and during breeding mutations are taking place with the probability $p$. More detailed definition of the model dynamics is given below:

(a) Choose a site at random (the chosen site is denoted by $i$ ).

(b) Provided that $i$ is occupied by a prey (i.e., if $x_{i}=1$ or $x_{i}=3$ ) update the prey with the probability $r$. If at least one neighbor (say $j$ ) of the chosen site is not occupied by a prey (i.e., $x_{j}=0$ or $x_{j}=2$ ), the prey at the site $i$ produces an offspring and places it on an empty neighboring site (if there are more empty sites, one of them is chosen randomly). Otherwise (i.e., if there are no empty sites) the prey does not breed.

(c) Provided that $i$ is occupied by a predator (i.e., $x_{i}=2$ or $x_{i}=3$ ) update the predator with the probability $(1-r) m_{i}$, where $m_{i}$ is the size of the predator at site $i$. If the chosen site $i$ is occupied by a predator only $\left(x_{i}=2\right)$, it dies, i.e., the site becomes empty $\left(x_{i}=0\right)$. If there is also a prey there $\left(x_{i}=3\right)$, the predator consumes the prey (i.e., $x_{i}$ is set to 2$)$ and if possible, it places an offspring at an empty neighboring site. For a predator of the size $m_{i}$ it is possible to place an offspring at the site $j$ provided that $j$ is not occupied by a predator $\left(x_{j}=0\right.$ or $\left.x_{j}=1\right)$ or is occupied by a predator $\left(x_{j}=2\right.$ or $x_{j}=3$ ) but of a smaller size than $m_{i}$ (in such a case the smaller-size predator is replaced by an offspring of the larger-size predator). The offspring inherits its parent's size with the probability $1-p$ and with the probability $p$ it gets a new size that is drawn from a uniform distribution.

At first sight one can think that such a model describes an ecosystem with two trophic levels (preys and predators) and only with predators being equipped with evolutionary abilities, which would be of course highly unrealistic. Let us notice, however, that expansion of predators sometimes proceeds at the expense of smaller-size predators. Thus, predators themselves are involved in prey-predator-like interactions. Perhaps it would be more appropriate to consider unmutable preys as a renewable (at a finite rate) source of, e.g., energy, and predators as actual species involved in various prey-predator interactions and equipped with evolutionary abilities.

\section{$3 \quad$ Results}

To examine the behaviour of this model we used numerical simulations. Our results, shown in Figs 14 are obtained for $r=0.2$ but we expect (Lipowski, 2005) that the behaviour of the model should be qualitatively the same for any $r<0.27$ (a brief discussion of the behaviour of the model for $r>0.27$ is given at the end of this section). 

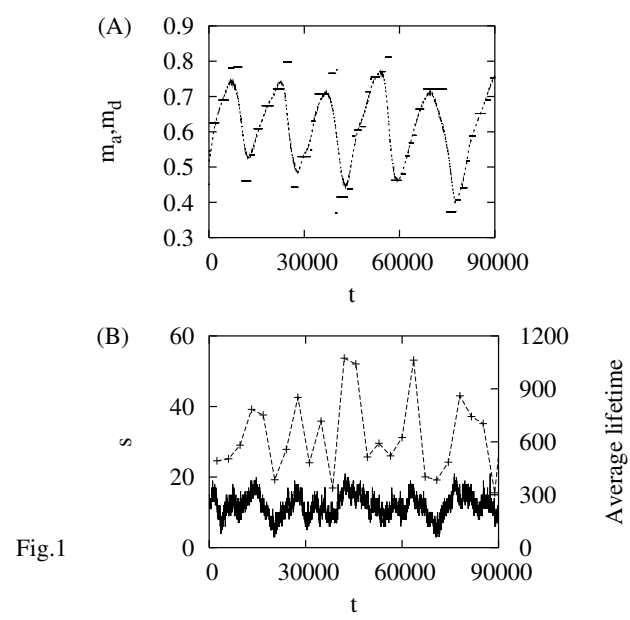

Figure 1: The results of numerical simulations $(N=500, \mathrm{p}=0.00001)$. Data on both panels are obtained from the same run. In our simulations a unit of time is defined as a single on average update of each site. (A) The time dependence of the average size $m_{a}$ (dashed line) and the size of the dominant species $m_{d}$ (short, horizontal intervals). (B) The number of species $s$ (continuous line) and the averaged lifetime of species $(+)$. After extinction the number of species drops 3-4 times. To reduce stochastic noise in the calculation of the average lifetime data are collected in time windows of the width $\Delta t=3000$.

\subsection{Oscillatory behaviour}

In Fig 1B one can see that, indeed, the number of species $s$ exhibits pronounced irregular oscillations. These oscillations are coupled with more regular oscillations of the averaged (over all predators) size $m_{a}$ (Fig 11A) and maxima of $s$ correspond approximately to minima of $m_{a}$ and vice versa. To have a better understanding of the behaviour of the model we also calculated the size $m_{d}$ of the dominant species (i.e., the predator species with the largest number of individuals) and the results are shown in Fig प1A.

These results indicate that the behaviour of our model can be described as follows: In a species-rich interval the size $m_{a}$ is typically quite low and there is an abundance of preys. In such a case predators of a large size are in a more favorable position (because a larger predator can replace a smaller predator) and as a result $m_{a}$ and $m_{d}$ increase. The process of increasing the size is gradual and involves a large number of species and is not related to a creation of a single (very-efficient) species, as suggested previously (Lipowski, 2005). The increased size $m$ implies a higher consumption rate and due to a finite recovery rate of preys the large-size species, that at this stage dominate the system, are running out of food. At first sight one might expect that further evolution will gradually reduce $m_{a}$ and $m_{d}$. Numerical results show, however (Fig 1A), that after reaching a local maximum, $m_{d}$ jumps to a very low value. This indicates that abrupt changes take place in the model after which large-size species are no longer dominant and vast majority of them become extinct. At the same time, however, a lot of new, mainly small-size species is created and that increases the diversity $s$ (although we do not suggest that this was really the cause, a succession of small mammals after large dinosaurs could be a vivid example of such a change). In such a way the system returns to the initial species-rich state. Such a cycle is also illustrated in Fig 2 that shows the distribution of size $m$ at various stages of the evolution ${ }^{1}$.

Gradual increase of size of species recalls the Cope's rule that states that species tend to

\footnotetext{
${ }^{1}$ Dynamics of the model is also illustrated with a Java applet available at: http:// spin.amu.edu.pl/lipowski/prey_pred.html
} 

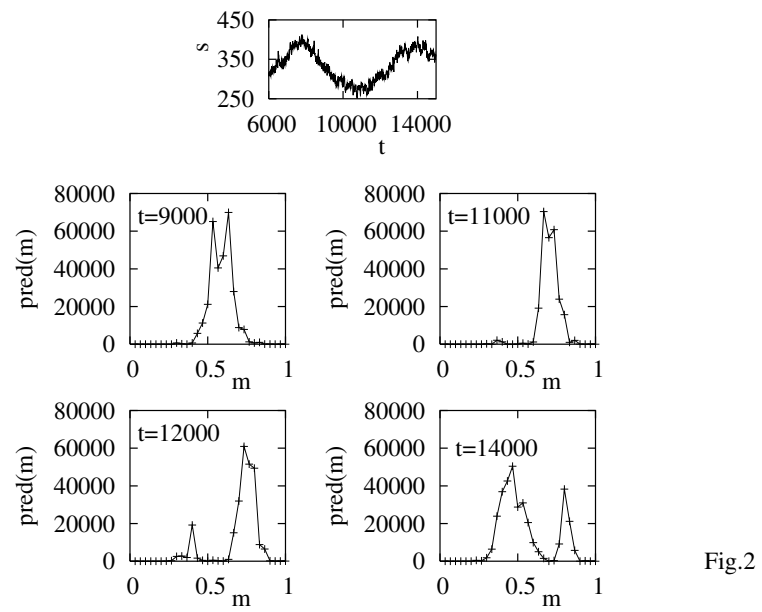

Figure 2: Four panels show distribution of sizes of species at various stages of evolutionary cycle $(N=1000, \mathrm{p}=0.0001)$. The upper panel shows the time dependence of the number of species $s$.

increase body size over geological time. This rule is not commonly accepted among paleontologists and evolutionists and was questioned on various grounds (Stanley, 1973). However, recent studies of fossil records of mammal species are consistent with this rule (Alroy, 1998; Van Valkenburgh et al., 2004). Perhaps our model could suggests a way to obtain a theoretical justification of this rule.

From the above description, it is expected that the periodicity of such a cycle increases when the mutation rate $p$ decreases, and such a behaviour is confirmed with more detailed calculations (Lipowski, 2005). In particular, already for $p=10^{-5}$ the estimated (Lipowski, 2005) periodicity of oscillations in our model is approximately 1000 times larger than that of the Lotka-Volterra oscillations in the corresponding single-predator system. It shows that the oscillations in our model are indeed long-period and, perhaps for smaller $p$, on a timescale close to $26 \mathrm{My}$.

Although very complicated, in principle, it should be possible to estimate the value of the mutation probability $p$ from the mutational properties of living species. Let us notice that in our model mutations produce an individual that might be substantially different from its parent. In Nature, this is typically the result of many cumulative mutations and thus we expect that $p$ is indeed a very small quantity. Actually, $p$ should be considered rather as a parameter related with the speed of morphological and speciation processes that are known to be typically very slow (Gingerich, 1983). Perhaps a modification of the mutation mechanism where a new species will be only a small modification of its parental species could be more suitable for comparison with living species, but it might require longer calculations. Alternatively, one can try to estimate the parameters $p, N$, and $r$ (or at least their ratios) by matching the behaviour of our model with some characteristics of the ecosystem such as the period of oscillations (26My), fraction of extinct species during a mass extinction or the average lifetime of species as compared with the periodicity of mass extinctions.

The oscillatory behaviour sets probably the largest timescale in our model. However, on the shorter timescale some characteristics, such as, e.g., the number of species, exhibit strong fluctuations (Fig. 1B). On such a timescale some distributions might be very broad and resemble power-law distributions. Indeed, such a behaviour was demonstrated for the distribution of lifetimes of species in our model (Lipowski, 2005).

The increase of the size of species in our model resembles the fitness-increasing evolution in the real ecosystem. It is tempting to consider present-day large mammals as highly adapted dominant species and, in the context of our model, located perhaps close to the local maximum 


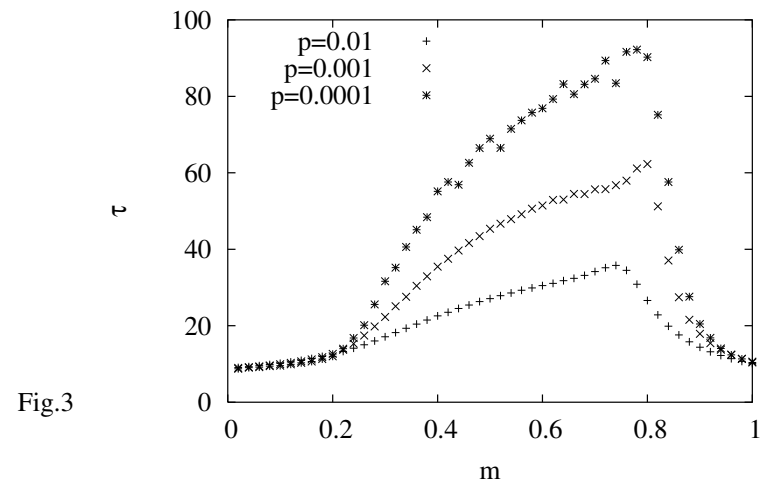

Figure 3: The average lifetime of species $\tau$ as a function of the size $m(N=500)$.

in the fitness space (as in Fig 1A). If so, then according to our model, the next dominant species most likely will be a small-size species that at the moment might not even exist. Its dominance will be possible due to drastic and inevitable changes of our ecosystem. Putting aside the validity of our model, such a scenario does not seem unlikely.

\subsection{Longevity of species}

An analysis of palaeontological data (Miller \& Foote, 2003) shows that the longevity is larger for species created after mass extinctions than for other species. To compare such a result with the predictions of our model, we calculated the average lifetime of species. It turns out, however, that important contributions to this quantity are coming from short-living species and their lifetime is essentially independent on the evolutionary phase at which they are born. To reduce this effect we took into account only the species that lived longer than a given threshold, which we set equal to 30 . Fossils of species of short lifetime are rather scarce and palaeontological data also reflect a similar bias toward long-lifetime species. The obtained results are shown in Fig 1B. Although still strongly fluctuating, they clearly show that the lifetime is correlated with the global evolution of the ecosystem and they qualitatively agree with palaeontological data. In particular, the maximum lifetime appears for species born shortly after a large and abrupt decrease of the size of the dominant species (crash). Apparently, species created at this time find most favourable conditions while the worst conditions exist shortly before a crash. Again using the analogy with the real ecosystem and humans, the model predicts (not counter-intuitively) that species created during our dominance will have a rather short lifetime.

For a species to have a very small size $m$ is usually a disadvantage since such a species will loose in competition with other species. On the other hand, a large size implies a high consumption rate and such a species might suffer from lack of food. It means that a lifetime of a species as a function of $m$ should have a maximum at a certain intermediate value and numerical calculations confirm such a behaviour (see Fig 3). Some data on distribution of sizes in Pleistocene and Recent molluscan faunas do show some maximum (Jablonsky et al., 2003) but a more detailed comparison cannot be done yet.

As our last result, we present the calculation of the average population size of species of a given lifetime (Fig (4). Although all the curves look qualitatively similar, one can notice a small difference between short- and long-lifetime species. This difference is better seen on the rescaled plot (Fig 4B). This data suggest that population sizes for species of a lifetime much shorter than the periodicity of extinctions (which in this case (Lipowski, 2005) is around 3000) after rescaling 


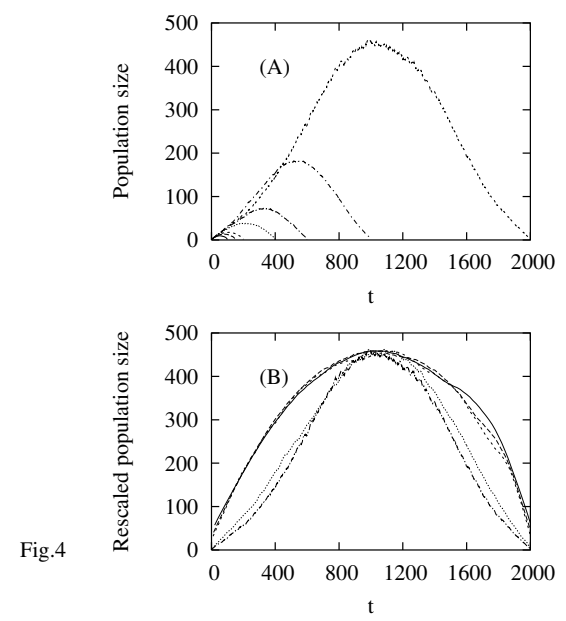

Figure 4: The analysis of the time dependence of the population size. (A) The average population size of species with a given lifetime $(p=0.001, N=500)$. (B) Some data from panel (A) rescaled (i.e., multiplied by some factors in both directions) in such a way that the lifetime and the maximal population size overlap. For species with the lifetime equal to 100, 150, and 200, the rescaled population sizes nearly overlap. Some deviations from the overlapping data can be seen for the lifetime 1000 and 2000.

fall into a single curve. For species of a lifetime comparable or larger than the periodicity of extinctions the data will deviate from such a universal curve. Although we are not aware of any palaeontological data of this kind, a comparison could provide an interesting test of our model.

\subsection{Unique code and the emergence of a multi-species ecosystem}

All living cells use the same code that is responsible for the transcription of information from DNA to proteins (Orgel, 1992; Szathmáry, 1999). It suggests that at a certain point of evolution of life on Earth a replicator that invented this apparently effective mechanism was able to eliminate replicators of all other species (if they existed) and establish, at least for a short time, a single-species ecosystem. Although this process is still to a large extent mysterious, one expects that subsequent evolution of these successful replicators leads to their differentiation and proliferation of species. In such a way the ecosystem shifted from a single- to multi-species one (Lipowski, 2000).

It seems to us that the present model might provide some insight into this problem. As we have already mentioned, the oscillatory behaviour appears in our model only for the relative update rate $r<0.27$. When preys reproduce faster $(r>0.27)$, a different behaviour can be seen (Lipowski, 2005) and the model reaches a steady state with almost all predators belonging to the same species with the size $m$ close to 1 . Only from time to time a new species is created with even larger $m$ and a change of the dominant species might take place. In our opinion, it is possible that at the very early period of evolution of life on Earth, the ecosystem resembled the case $r>0.27$. This is because at that time substrates ('preys') were renewable faster than primitive replicators ('predators') could use them. If so, every invention of the increase of the efficiency ('size') could invade the entire system. In particular, the invention of the coding mechanism could spread over the entire system. A further evolution increased the efficiency of predators and that effectively shifted the (single-species) ecosystem toward the $r<0.27$ (multi-species, oscillatory) regime. 


\section{Conclusions}

In the present paper we examined a spatially extended multi-species prey-predator model. In a certain regime in this model densities of preys and predators as well as the number of species show long-term oscillations, even though the dynamics of the model is not exposed to any external periodic forcing. It suggests that the oscillatory behaviour of the Earth ecosystem predicted by Raup and Sepkoski could be simply a natural feature of its dynamics and not the result of an external factor. Some predictions of our model such as the lifetime of species or the time dependence of their population sizes might be testable against palaeontological data. The prediction that a lifetime of species depends on the evolutionary stage at which it was created, that qualitatively agrees with fossil data (Miller \& Foote, 2003), suggests that a further study of this model would be desirable.

Certainly, our model is based on some restrictive assumptions that drastically simplifies the complexity of the real ecosystem. We hope, however, that it includes some of its important ingredients: replication, mutation, and competition for resources (food and space). As an outcome, the model shows that typically there is no equilibrium-like solution and the ecosystem remains in an evolutionary cycle. The model does not include geographical barriers but let us notice that palaeontological data that suggest the periodicity of mass extinctions are based only on marine fossils (Rohde \& Muller, 2005). More realistic versions should take into account additional trophic levels, gradual mutations, or sexual reproduction. One should also notice that the palaeontological data are mainly at a genus, and not species level. It would be desirable to check whether the behaviour of our model is in some sense generic or it is merely a consequence of its specific assumptions. An interesting possibility in this respect could be to recast our model in terms of Lotka-Volterra like equations and use the methodology of adaptive dynamics developed by Dieckmann et al. (Dieckmann et al., 1995).

Of course, the real ecosystem was and is exposed to a number of external factors such impacts of astronomical objects, volcanism or climate changes. Certainly, they affect the dynamics of an ecosystem and contribute to the stochasticity of fossil data. Filtering out these factors and checking whether the main evolutionary rhythm is indeed set by the ecosystem itself, as suggested in the present paper, is certainly a difficult task but maybe worth an effort.

\section{Acknowledgements}

The research grant 1 P03B 01427 from KBN is gratefully acknowledged. We thank Department of Physics of the University of Aveiro (Portugal) for giving us access to computing facilities.

\section{REFERENCES}

Alroy, J., 1998. Cope's Rule and the Dynamics of Body Mass Evolution in North American Fossil Mammals, Science 280, 731-734.

Bak, P \& Sneppen, K., 1993. Punctuated equilibrium and criticality in a simple model of evolution, Phys. Rev. Lett. 71, 4083-4086.

Caldarelli, G., Higgs, P. G. \& McKane, A. J., 1998. Modelling coevolution in multispecies communities, J. Theor. Biol. 193, 345-358.

Chowdhury, D., Stauffer, D. \& Kunwar, A., 2003. Unification of Small and Large Time Scales for Biological Evolution: Deviations from Power Law, Phys. Rev. Lett. 90, 068101-068105. 
Coppex, F., Droz, M. \& Lipowski, A., 2004. Extinction dynamics of Lotka-Volterra ecosystems on evolving networks, Phys. Rev. E 69, 061901-061907.

Davis, M., Hut, P. \& Muller, R. M., 1984. Extinction of species by periodic comet showers, Nature 308, 715-717.

Dieckmann, U., Marrow, P. \& Law, R., 1995. Evolutionary Cycling in Predator-Prey Interactions: Population Dynamics and the Red Queen, J. Theor. Biol. 176, 91-102.

Dieckmann, U., Law, R. \& Metz, J. A. J., 2000. The Geometry of Ecological Interactions: Simplifying Spatial Complexity, (Cambridge University Press).

Fox, W. T., 1987. Harmonic analysis of periodic extinctions, Paleobiology 13, 257-271.

Gingerich, P. D., 1983. Rates of Evolution: Effects of Time and Temporal Scaling, Science 222, 159-161.

Jablonski, D., Roy K. \& Valentine, J. W., 2003. Evolutionary macroecology and the fossil record. In: T.M. Blackburn and K. J. Gaston, eds., Macroecology: Concepts and Consequences, 368-390 (Oxford: Blackwell Science).

Lipowski A., 1999. Oscillatory behaviour in a lattice prey-predator system, Phys. Rev. E 60, 5179-5184.

Lipowski A., 2000. Multiplicity of species in some replicative systems, Phys. Rev. E 61, 30093014.

Lipowski, A., 2005. Periodicity of extinctions without an extraterrestrial cause, Phys. Rev. E 71, 052902-052905.

Lipowski A. \& Lipowska, D., 2000. Nonequilibrium phase transition in a prey-predator system. Physica A 276, 456-464.

Miller, A. I. \& Foote, M., 2003. Increased Longevities of Post-Paleozoic Marine Genera After Mass Extinctions, Science 302, 1030-1032.

Murray, J. D., 1989. Mathematical Biology, (Springer, Berlin).

Newman, M. E. J \& Palmer, R. G., 2003. Modelling Extinction, (Oxford University Press, New York).

Orgel, L. E., 1992. Molecular replication, Nature 358, 203-209.

Patterson, C. \& Smith, A. B., 1989. Periodicity in extinction: the role of the systematics, Ecology 70, 802-811.

Pȩkalski, A., 2004. A short guide to predator-prey lattice models, Comput. Sci. Eng. 6, 62-66.

Pimm, S. L., 2002. Food Webs (University of Chicago Press, Chicago).

Plotnick, R. E. \& McKinney, M. L., 1993. Ecosystem organization and extinction dynamics, Palaios 8, 202-212.

Plotnick, R. E. \& Sepkoski, J. J., 2001. A multiplicative multifractal model for originations and extinctions, Paleobiology 27, 126-139.

Prokoph, A., Fowler, A. D. \& Patterson, R. T., 2000. Evidence for periodicity and nonlinearity in a high-resolution fossil record of long-term evolution, Geology 28, 867-870. 
Rampino, M. R. \& Stothers, R. B., 1984. Terrestrial mass extinctions, cometary impacts and the Sun's motion perpendicular to the galactic plane, Nature 308, 709-712.

Raup, D. M. \& Sepkoski, J. J., 1984. Periodicities of extinctions in the geologic past, Proc. Natl. Acad. Sci. 81, 801-805.

Rohde, R. A. \& Muller, R. A., 2005. Cycles in fossil diversity, Nature 434, 208-210.

Sato, K., N. Yoshida \& Konno, N., 2002. Parity law for population dynamics of N-species with cyclic advantage competitions, Appl. Math. Comput. 126, 255-270.

Solé, R. V. \& Manrubia, S. C., 1999. Extinction and self-organized criticality in a model of large-scale evolution, Phys. Rev. E 54, R42-R45.

Stanley, S. M, 1973. An explanation for Cope's rule, Evolution 27, 1-26.

Stanley, S. M, 1990. Delayed recovery and the spacing of major extinctions, Paleobiology 16, 401-414.

Stothers, R. B., 1986. Periodicity of the Earth's magnetic reversals, Nature 322, 444-446.

Stothers, R. B., 1993. Flood basalts and extinction events, Geophys. Res. Lett. 20, 1399-1402.

Szathmáry, E., 1999. The origin of the genetic code Trends in Genetics 15, 223-229.

Van Valkenburgh, B., Wang, X. \& Damuth, J., 2004. Cope's Rule, Hypercarnivory, and Extinction in North American Canids, Science 306, 101-104. 